\title{
CEREBROVASCULAR COMPLICATIONS IN PROLONGED ANTIHYPERTENSIVE THERAPY IN MEN WITH ESSENTIAL HYPERTENSION
}

\author{
$\underline{\text { R.Aliyev }}^{1}$, F.Gasimova ${ }^{2}$, F.Gasimova ${ }^{2}$ \\ ${ }^{1}$ Azerbaijan State Advanced Training Institute for Doctors named after A.Aliyev, \\ Department of Neurology and clinical neurophysiology, Baku, Azerbaijan; \\ ${ }^{2}$ Azerbaijan State Advanced Training Institute for Doctors named after A.Aliyev, \\ Department of Therapy, Baku, Azerbaijan
}

Background and Aims. Arterial hypertension (AH) ranks first in the structure of mortality from cardiovascular diseases and is a risk factor for cerebrovascular diseases, including stroke. Obviously, the role of antihypertensive therapy (AHT) in prevention of cerebrovascular complications of hypertension is beyond doubt. The aim of this study was to observe the rate of cerebrovascular complications with longterm AHT in patients with essential hypertension.

Method. 225 men aged 30-59 years with AH were examined. All patients took various antihypertensive drugs, both in the form of monotherapy, and their various combinations. All patients were examined by a neurologist. Before entering to the study, it was not found any disorders of nervous system associated with $\mathrm{AH}$.

Results. With prolonged AHT, complications of hypertension were observed in 29 patients $(21.1 \%)$ at different treatment times, 3 of which $(2.2 \%)$ died from other causes. In 12 patients $(8.7 \%)$ equally fatal and nonfatal cerebrovascular complications were detected. Against the background of continuous treatment, the complication of hypertension arose on average $8.6 \pm 4.5$ years. In $75 \%$ of cases, betablockers were used in 9 of 12 patients with cerebrovascular complications with AHT, 5 of them developed a fatal stroke (55.6\%), and 4 had nonfatal (44.4\%).

Conclusion. It was found that fatal and nonfatal cerebrovascular complications appeared mainly in patients taking betablockers, both in the form of monotherapy, and their combination. Long-term AHT allows not only to adequately control of blood pressure, but also to reduce the frequency of its complications, in particular, ischemic strokes, while long-term treatment with betablockers does not prevent their development.

Keywords: arterial hypertension, antihypertensive therapy, risk factors, cerebrovascular complications, ischemic stroke. 